Meta

Journal des traducteurs

Translators' Journal

\title{
Las competencias del gestor de proyectos de traducción: análisis de un corpus de anuncios de trabajo
}

\section{Cristina Plaza-Lara}

Volume 63, numéro 2, août 2018

URI : https://id.erudit.org/iderudit/1055150ar

DOI : https://doi.org/10.7202/1055150ar

Aller au sommaire du numéro

\section{Éditeur(s)}

Les Presses de l’Université de Montréal

ISSN

0026-0452 (imprimé)

1492-1421 (numérique)

Découvrir la revue

Citer cet article

Plaza-Lara, C. (2018). Las competencias del gestor de proyectos de traducción: análisis de un corpus de anuncios de trabajo. Meta, 63(2), 510-531. https://doi.org/10.7202/1055150ar
Résumé de l'article

$\mathrm{Au}$ cours des dernières décennies, le marché de la traduction a connu d'importants bouleversements sous l'effet de la mondialisation. L'entrée en scène de différents acteurs a imposé une gestion par projet comme mode d'organisation privilégié du travail. Bien qu'il s'agisse d'une pratique courante dans d'autres domaines, très peu de recherches se sont penchées sur la gestion de projet dans notre discipline: en effet, le caractère unique et éphémère des projets de traduction et les caractéristiques du secteur rendent les conclusions difficiles. Ce constat s'accentue lorsque l'on prend en compte les compétences du chef de projet (PM). C'est pourquoi la présente étude expose les résultats de l'analyse d'un corpus d'offres d'emploi pour le poste de chef de projet de traduction, afin de saisir la manière dont les employeurs perçoivent les compétences des PM. Les 100 annonces qui composent ce corpus - interprétées grâce à une analyse de contenu - seront comparées avec les aptitudes décrites dans la bibliographie sur la gestion de projet et la traduction. L'objectif est de cerner les compétences d'un chef de projet dans ce domaine à partir des données recueillies.
Tous droits réservés C Les Presses de l’Université de Montréal, 2018
Ce document est protégé par la loi sur le droit d'auteur. L'utilisation des services d'Érudit (y compris la reproduction) est assujettie à sa politique d'utilisation que vous pouvez consulter en ligne.

https://apropos.erudit.org/fr/usagers/politique-dutilisation/ 


\title{
Las competencias del gestor de proyectos de traducción: análisis de un corpus de anuncios de trabajo
}

\author{
CRISTINA PLAZA-LARA \\ Universidad de Málaga, Málaga, España \\ cplaza@uma.es
}

\section{RÉSUMÉ}

Au cours des dernières décennies, le marché de la traduction a connu d'importants bouleversements sous l'effet de la mondialisation. L'entrée en scène de différents acteurs a imposé une gestion par projet comme mode d'organisation privilégié du travail. Bien qu'il s'agisse d'une pratique courante dans d'autres domaines, très peu de recherches se sont penchées sur la gestion de projet dans notre discipline: en effet, le caractère unique et éphémère des projets de traduction et les caractéristiques du secteur rendent les conclusions difficiles. Ce constat s'accentue lorsque l'on prend en compte les compétences du chef de projet (PM). C'est pourquoi la présente étude expose les résultats de l'analyse d'un corpus d'offres d'emploi pour le poste de chef de projet de traduction, afin de saisir la manière dont les employeurs perçoivent les compétences des PM. Les 100 annonces qui composent ce corpus - interprétées grâce à une analyse de contenu - seront comparées avec les aptitudes décrites dans la bibliographie sur la gestion de projet et la traduction. L'objectif est de cerner les compétences d'un chef de projet dans ce domaine à partir des données recueillies.

\begin{abstract}
In recent decades, the translation market has undergone notable changes as a result of the process of globalization. Several agents enter into play in this scenario, which has led to the need for project management as a method of organizing work. In spite of the fact that this method has been used in other sectors, research in our discipline has paid scant attention to project management, in part because the one-off and temporary nature of the projects and the characteristics of the sector make it difficult to draw conclusions. This is emphasized further when looking in detail at the competences of a project manager (PM). To address this gap in the research, in the present study the results of an analysis of a corpus of job advertisements directed at translation project managers are presented, in order to understand how employers describe the competencies required of a PM. The corpus is made up of a total of 100 advertisements, which were analyzed using the technique of content analysis, and compared with the competences described in the translation and project management literature. The data extracted can be used to define a project manager's competences in this area.
\end{abstract}

\section{RESUMEN}

En las últimas décadas, el mercado de la traducción ha experimentado notables cambios impulsados por el proceso de globalización. En este escenario entran en juego diferentes agentes, lo que ha obligado a establecer la gestión por proyectos como modo de organización del trabajo. A pesar de contar con tradición en otros sectores, las investigaciones en nuestra disciplina apenas han prestado atención a la gestión de proyectos, ya que la naturaleza única y temporal de estos y las características del sector dificultan extraer conclusiones. Todo ello se acentúa al profundizar en las competencias del gestor de proyectos o PM. Por este motivo, en el presente trabajo, se exponen los resultados de 
un análisis de un corpus de anuncios de trabajo dirigidos a gestores de proyectos de traducción, para comprender cómo describen los empleadores las competencias de los PM. El corpus está compuesto por un total de 100 anuncios que, analizados mediante la técnica de análisis de contenidos, se compararán con las competencias descritas en la bibliografía de gestión de proyectos y de traducción. A partir de los datos extraídos, se pretende acotar las competencias de un gestor de proyectos en este ámbito.

\section{MOTS-CLÉS/KEYWORDS/PALABRAS CLAVE}

gestion de projet, définition de compétences, corpus d'offres d'emploi, analyse de contenu, métiers de la traduction

project management, definition of competencies, corpus of job advertisements, content analysis, translation-related professions

gestión de proyectos, definición de competencias, corpus de anuncios de trabajo, análisis de contenidos, profesiones relacionadas con la traducción

\section{Introducción}

En traductología, se empiezan a escuchar voces de autores como Chesterman (2009) o Pym (2009) que abogan por describir el comportamiento de los traductores, más que de las traducciones y hablan de Translator Studies y no de Translation Studies (Ferrer Simó 2016: 2). Por su parte, el sector de la traducción, que ha crecido exponencialmente en las últimas décadas, se caracteriza por ser un mercado «highly projectized, globalized, diverse, and fast-moving» (Dunne 2013: 2). Este escenario obliga a investigadores de la disciplina a prestar atención a los nuevos perfiles profesionales que han aparecido en torno a la figura del traductor con el fin de obtener una perspectiva global e integradora.

La gestión de proyectos se define como la aplicación de un conjunto de conocimientos, habilidades, herramientas y técnicas a las tareas del proyecto para cumplir los objetivos establecidos (Project Management Institute - $\mathrm{PMI}^{1}$ 2002/2007: 75). El gestor de proyectos (GP, según la norma ISO $17100^{2}$ ), comúnmente conocido por su denominación en inglés project manager (PM, sigla utilizada en este artículo dado su uso extendido en el sector), es la persona encargada de que se cumplan dichos objetivos. Aunque la organización por proyectos cuenta con una larga tradición en sectores como la ingeniería, el reciente desarrollo del mercado de la traducción ha impuesto la aplicación de sus fundamentos al modo de trabajo de los profesionales de nuestro campo.

Sin embargo, la profesión del PM se considera en ocasiones una profesión accidental, una transición en la que normalmente confluyen experiencia y necesidades organizativas. Si bien existen gestores de proyectos sin experiencia previa en traducción, la evolución habitual en muchas empresas suele ser el paso de traductor/revisor a gestor de proyectos. Esto no significa que cualquier traductor pueda desempeñar tareas de gestión de proyectos; igualmente, el nuevo PM precisará de ciertos conocimientos para habituarse a las diferentes responsabilidades.

Pese a que muchos de los principios que se aplican en la gestión de proyectos de diferentes disciplinas son extrapolables a la traducción, la naturaleza temporal y única de los proyectos (PMI 1996/2013: 5) y las características específicas del sector dificultan extraer conclusiones sobre las competencias del gestor de proyectos de traducción. Por este motivo, en este estudio se parte de un enfoque sistemático basado en el 
análisis de un corpus de anuncios de trabajo a escala global con el fin de comprender cómo describen los empleadores las competencias de los PM. Dichas competencias se compararán con las competencias descritas en la bibliografía existente, tanto de gestión de proyectos como de traducción. El objetivo principal es sentar la base para futuras investigaciones que permitan crear un modelo de competencias del gestor de proyectos de traducción.

El artículo se divide en tres partes principales. En primer lugar, se delimita el marco teórico tanto de la gestión de proyectos como disciplina independiente, como de la gestión de proyectos en traducción, haciendo especial énfasis en la definición de las competencias. Posteriormente, se aborda el diseño del corpus y sus características, y la metodología empleada para su elaboración, así como para el subsiguiente análisis de los datos. Por último, se exponen las conclusiones extraídas de dicho análisis.

\section{Marco teórico: delimitación de las competencias para la gestión de proyectos de traducción}

\subsection{La gestión de proyectos como disciplina independiente}

La gestión de proyectos se empezó a aplicar en el ámbito empresarial de forma más sistemática a partir de los años 30 del siglo pasado (Way 2009: 133) y, desde entonces, se ha convertido en un modo de organización del trabajo de diferentes sectores a escala mundial. Si bien no constituye una práctica reciente, es bastante escasa la bibliografía existente al respecto (Hölzle 2010: 779; Matcha Abombo 2015: 308) o, como advierten Dunne y Dunne (2011: 8), en muchos casos se focaliza en campos que tradicionalmente se han servido de esta disciplina, como la construcción o la ingeniería. No obstante, su implantación como práctica profesional ha motivado la proliferación de organizaciones y asociaciones nacionales e internacionales, que fomentan la investigación en este ámbito y que formulan estándares aplicables a la gestión de proyectos. Mundialmente conocido es el Project Management Institute $(\mathrm{PMI})^{3}$, que cuenta con una sólida reputación desde su fundación en EE. UU. en el año 1969, aunque también destacan la suiza International Project Management Association (IPMA) ${ }^{4}$, organizada como una federación internacional, u otras a escala estatal, como la Association for Project Management ${ }^{5}$, del Reino Unido.

Entre las publicaciones más destacadas de estas organizaciones cabe mencionar A Guide to the Project Management Body of Knowledge, también denominada PMBOK ${ }^{\oplus}$ Guide (PMI 1996/2013). Desde la primera edición en 1983 del documento técnico que luego daría lugar a la $P M B O K^{\oplus}$ Guide, esta organización pretende documentar y estandarizar las prácticas comúnmente aceptadas en gestión de proyectos. En relación con las competencias, tema central de este artículo, el mismo instituto ha publicado el Project Management Competency Development (PMCD) Framework (PMI 2002/2007 $)$ e igualmente oferta diferentes certificaciones a quienes se dedican a la gestión de proyectos (dos ejemplos son la certificación $\mathrm{PMP}^{\circledR}$ o la CAPM ${ }^{\circledast}$ ). En torno a las competencias es preciso nombrar también el modelo ICB (IPMA Competence Baseline) de la International Project Management Association, que divide las competencias en contextuales, técnicas y comportamentales. Para la presente investigación se tomará como referencia el modelo PMCD Framework (PMI 
2002/2007) dada su vinculación con la $P M B O K^{\oplus}$ Guide, referente en el sector, y las certificaciones ofrecidas por el PMI.

El PMCD Framework define la competencia del gestor de proyectos como la capacidad de aplicar el conocimiento sobre gestión de proyectos y de adecuar el comportamiento para aumentar las probabilidades de entregar proyectos que cumplan con los requisitos de las partes interesadas (PMI 2002/2007: 2). Al igual que en la $P M B O K^{\oplus}$ Guide, esta competencia se compone de tres dimensiones:

- conocimiento: lo que sabe el gestor de proyectos sobre la aplicación de procesos, herramientas y técnicas;

- desempeño: cómo se aplica ese conocimiento para cumplir los requisitos del proyecto;

- personal: cómo se comporta el gestor de proyectos (actitudes y características personales) (PMI 2002/2007: 2).

La primera dimensión se detalla en las especificaciones para la certificación $\mathrm{PMP}^{\circledast}$, aunque se puede extraer un resumen de las áreas de conocimientos de la $P M B O K^{\oplus}$ Guide: integración del proyecto, alcance del proyecto, duración del proyecto, costes del proyecto, calidad del proyecto, recursos humanos involucrados en el proyecto, comunicación durante el proyecto, riesgos del proyecto y adquisiciones del proyecto (PMI 1996/2013). Como se puede inferir de la siguiente cita, se considera que determinadas áreas tienen mayor impacto en la consecución del proyecto:

however, in a recent study by Zwikael (2009), of 783 project managers from different countries and industries, it was found that the Knowledge Areas from the PMBOK Guide (PMI, 2002/2007) with the greatest impact on project success were Project Time Management, Project Risk Management, Project Scope Management, and Project Human Resource Management (Ahsan, Ho, et al. 2013: 39).

En lo que concierne a las dimensiones de desempeño y personal descritas en el PMCD Framework, cabe destacar que este modelo presenta una minuciosa descripción de ambas de acuerdo con la siguiente estructura:

FIGURA 1

Estructura de las dimensiones de la competencia del gestor de proyectos según el PMCD Framework (imagen de creación propia)

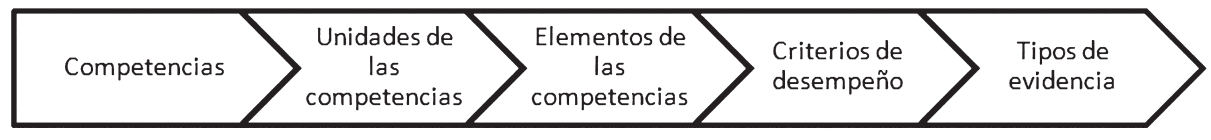

En los diferentes niveles de definición se especifican los requisitos que un PM debe cumplir para demostrar su competencia en gestión de proyectos y se definen criterios de rendimiento para evaluar dicha competencia. A continuación, se recogen las unidades para las dimensiones de desempeño y personal. 
CUADRO 1

Unidades de las dimensiones de desempeño y personal de la competencia del gestor de proyectos (PMI 2002/2007)

\begin{tabular}{|l|l|}
\hline Performance Competencies & Personal Competencies \\
\hline 1.0 Initiating a Project & 6.0 Communicating \\
\hline 2.0 Planning a Project & 7.0 Leading \\
\hline 3.0 Executing a Project & 7.0 Managing \\
\hline 4.0 Monitoring \& Controlling a Project & 9.0 Cognitive Ability \\
\hline 5.0 Closing a Project & 10.0 Effectiveness \\
\hline & 11.0 Professionalism \\
\hline
\end{tabular}

\subsection{La gestión de proyectos de traducción}

Pese a que la gestión de proyectos de traducción constituye uno de los pilares fundamentales en el sector de proveedores de servicios lingüísticos, «it has been largely overlooked as an object of scholarly inquiry and critical pedagogical reflection in the field of translation studies» (Dunne y Dunne 2011: 6). La bibliografía existente en este ámbito es bastante reciente y solo en trabajos publicados a partir del año 2010 se empieza a abordar con detenimiento esta nueva salida profesional. Este hecho se acentúa al profundizar en las competencias del gestor de proyectos, donde apenas se encuentran investigaciones teóricas o empíricas. Al margen de esta falta de definición de competencias, la gestión de proyectos ha empezado a incorporarse en los contenidos del grado (véanse, por ejemplo, las propuestas de Way 2009, Flores Acuña 2012 o Plaza-Lara 2016) y cada vez son más los estudios de máster centrados en esta especialidad $^{7}$ o que ofertan bloques específicos.

Con el fin de acotar el marco teórico, se mencionarán a continuación algunas investigaciones que consideramos relevantes sobre esta materia. Durante la primera década de este milenio, es posible encontrar capítulos de libro (Esselink 2000; Gouadec 2002; 2007; 2009) o artículos de revista (Arevalillo Doval 2002; Rico Pérez 2002; Torres Hostench 2002; Stoeller 2004; ; Goaudec 2005) que giran en torno a las distintas fases de la gestión de proyectos y a las tareas que debe desarrollar un PM. En el año 2004, la revista Multilingual Computing \& Technology ${ }^{9}$ prepara un suplemento en exclusiva sobre este campo. En el año 2010, Matis publica un libro en el que ofrece una visión amplia y detallada de la gestión de proyectos, fases, factores que intervienen y procedimientos más habituales. Esta guía puede constituir una herramienta de apoyo para aquellos que se inician en este ámbito. Un año más tarde se publica el primer volumen de contribuciones sobre gestión de proyectos de traducción y localización, de la mano de Dunne y Dunne (2011). Este volumen recoge un conjunto de trabajos que abordan diferentes temáticas, que van desde la calidad hasta el software de gestión de proyectos, pasando por factores clave como son los plazos y la gestión de recursos humanos. La misma Elena Dunne defiende en el año 2013 su tesis doctoral sobre la gestión de riesgos.

En el último lustro se han defendido en España varias tesis doctorales sobre esta temática. Por un lado, cabría citar la tesis de Arevalillo Doval (2016), en torno a la calidad en la gestión de proyectos, y la de Ferrer Simó (2016), sobre la gestión de proyectos de traducción audiovisual. En el presente artículo, queremos destacar el trabajo de Matcha Abombo (2014), que aborda la implementación de la gestión de 
proyectos en los estudios de Traducción e Interpretación. Su orientación hacia la didáctica obliga al autor a dedicar un capítulo a las competencias del gestor de proyectos. Para tal fin, toma como punto de partida la propuesta del PMCD Framework y distingue entre competencia de planificación, organización, ejecución, y seguimiento y control (Matcha Abombo 2014: 241).

Por otro lado, resulta fundamental aludir a la norma ISO 17100 (2015: 17), que entre las tareas del gestor de proyectos incluye las siguientes:

a) identificar los requisitos y especificaciones fundamentales del proyecto de traducción durante el proceso de preproducción, y seguir los procedimientos y especificaciones a lo largo de toda su producción;

b) supervisar y controlar el proceso de preparación del proyecto de traducción;

c) asignar a un traductor o traductores competentes para el proyecto de traducción;

d) asignar a un revisor o revisores competentes;

e) distribuir la información, emitir las instrucciones relacionadas con la asignación y gestionar el proyecto de traducción para todas las partes involucradas;

f) controlar el cumplimiento de planificaciones y plazos acordados;

g) comunicar los cambios que se produzcan en las especificaciones del proyecto, cuando proceda;

h) supervisar la conformidad constante con el acuerdo entre el cliente y el PST, las especificaciones del proyecto $y$, cuando sea necesario, comunicarse con todas las partes involucradas en el proyecto, incluido el cliente;

i) garantizar que se responde a las consultas sobre la traducción y de otro tipo;

j) gestionar y administrar los comentarios del cliente;

k) verificar el cumplimiento de las especificaciones del servicio de traducción antes de la aprobación del contenido de la lengua de destino y de la autorización de su entrega al cliente;

1) prestar el servicio.

Además, añade tareas adicionales como nombrar un corrector de concepto, implantar acciones correctoras, controlar el presupuesto, preparar la factura y realizar otras tareas acordadas con el cliente. Aunque en esta norma no se incluyen todas las competencias detalladas en la publicación del PMI, basta con comparar estas tareas con las competencias descritas en el PMCD Framework para establecer ciertas similitudes entre ellos.

En contraposición a esta «indefinición» de las competencias del gestor de proyectos de traducción, la investigación en traductología se ha interesado por las competencias del traductor casi desde los orígenes de la disciplina. Son muchos los modelos que intentan definir sus componentes y que parten de diferentes elementos de categorización (Calvo Encinas 2010: 62), si bien destacan las investigaciones de Hurtado Albir y su grupo de investigación, PACTE, de Kelly (2005) y del European Master's in Translation ${ }^{10}$. Para el presente artículo, se tomará como referencia el modelo propuesto por Hurtado Albir y PACTE, dada su sólida trayectoria en investigación empírico-experimental sobre la competencia traductora.

- Bilingual sub-competence. Predominantly procedural knowledge required to communicate in two languages. It includes the specific ability of controlling interference when switching between two languages. It comprises pragmatic, sociolinguistic, textual, grammatical and lexical knowledge in the two languages. [...]

- Extralinguistic sub-competence. Predominantly declarative knowledge, both implicit and explicit, about the world in general and special areas. It comprises: 
(1) bicultural knowledge (about the source and target cultures); (2) encyclopaedic knowledge (about the world in general); (3) subject knowledge (field-specific).

- Knowledge of Translation sub-competence. Predominantly declarative knowledge, both implicit and explicit, about what translation is and aspects of the profession. It comprises: (1) knowledge about how translation functions: translation units, processes required, methods and procedures used (strategies and techniques), and types of problems; (2) knowledge related to professional translation practice: the work market, types of translation briefs, target audiences, etc.

- Instrumental sub-competence. Predominantly procedural knowledge related to the use of documentation resources and information and communication technologies applied to translation: dictionaries of all kinds, encyclopaedias, grammars, style books, parallel texts, electronic corpora, search engines, etc.

- Strategic sub-competence. Procedural knowledge to guarantee the efficiency of the translation process and solve problems encountered. This is an essential subcompetence that affects all the others since it creates links between the different subcompetences as it controls the translation process. Its functions is: (1) to plan the process and carry out the translation project (selecting the most appropriate method); (2) to evaluate the process and the partial results obtained in relation to the final purpose; (3) to activate the different sub-competences and compensate for any shortcomings in them; (4) to identify translation problems and apply procedures to solve them.

- Psycho-physiological components. Different types of cognitive and attitudinal components and psycho-motor mechanisms. They include: (1) cognitive components such as memory, perception, attention and emotion; (2) attitudinal aspects such as intellectual curiosity, perseverance, rigour, critical spirit, knowledge about and confidence in one's own abilities, the ability to measure one's own abilities, motivation, etc.; (3) abilities such as creativity, logical reasoning, analysis and synthesis, etc. (Hurtado Albir 2017: 39-40).

Aunque este modelo de competencias hace referencia directa al perfil del traductor, como se señala en diferentes publicaciones la profesión del PM es una profesión accidental a la que se puede acceder con o sin conocimientos previos en traducción (Arevalillo Doval 2002: 13; Zouncourides-Lull 2011: 72; Ahsan, Ho, et al. 2013: 37). Compartimos la opinión de Arevalillo Doval sobre gestores de proyectos sin formación en traducción:

[e]n este caso, difícilmente podrán comprender los problemas del día a día de nuestro sector, en especial si tenemos en cuenta el desconocimiento habitual que los clientes tienen sobre la traducción y que puede derivar en solicitudes de trabajo irreales y problemáticas (Arevalillo Doval 2002: 13).

Por lo tanto, para poder esbozar un modelo de competencias para la gestión de proyectos en nuestro campo, se debe partir de las competencias del traductor y combinarlas con las del PM. En el presente trabajo partiremos de las competencias descritas por PACTE y por el PMCD Framework. Se considera que la subcompetencia estratégica y los componentes psicofisiológicos de PACTE se pueden fusionar al menos en parte con las dimensiones de desempeño y personal definidas por el $P M C D$ Framework. De esta forma, se podrá corroborar si el gestor de proyectos de traducción requiere de unas competencias distintas al traductor. El mismo PMI señala que, aunque sus competencias se pueden transferir de un campo a otro, es necesario desarrollar modelos específicos para cada sector (Ahsan, Ho, et al. 2013: 43). 


\section{Análisis de un corpus de anuncios de trabajo}

\subsection{Diseño del corpus y metodología de análisis}

Son varios los investigadores en traductología que han empleado anuncios de trabajo como herramienta de investigación. Por un lado, Bowker (2004) se encarga de analizar la demanda de traductores en Canadá entre los años 2000 y 2002. Para ello analiza un corpus compuesto por 301 anuncios de empleo, a fin de esbozar las características de la demanda en ese momento. Por su parte, Chan (2008) estudia un corpus de anuncios de trabajo del año 2006 para ver qué cualificaciones y experiencia se demandan. Igualmente, Gouadec (2007), a partir de los resultados de un estudio basado en anuncios de trabajo, presenta una lista de competencias del traductor profesional por orden de prioridad.

Los anuncios de trabajo son una herramienta de contratación básica que las organizaciones utilizan para dirigirse a los solicitantes de vacantes (Johnson, Winter, et al. 2008). Por este motivo y dada la falta de investigaciones en torno a las competencias del gestor de proyectos en traducción, el corpus creado para el presente estudio incluye un total de 100 anuncios de trabajo dirigidos a gestores de proyectos en el campo de la traducción. A partir de los datos proporcionados por dicho corpus, se pretende acotar las competencias de un gestor de proyectos en este ámbito. De esta forma, se adopta un enfoque inductivo que tiene como objetivo obtener conclusiones generales a partir de datos concretos.

Para la elaboración del corpus se ha recurrido únicamente a anuncios de trabajo publicados en Internet, ya que el mercado internacional en el que trabajan las empresas de traducción (o aquellas que requieren de estos servicios) obliga a publicar en línea la mayoría de las ofertas, que no llegarían a sus destinatarios si se publicaran únicamente en prensa. Se trata por tanto de un corpus en formato electrónico. Asimismo, dado el carácter global del mercado de la traducción, tampoco se han limitado los anuncios a una determinada zona geográfica, ni se han filtrado por ubicación para obtener representación de diferentes zonas. La fecha de publicación de los anuncios oscila entre mayo de 2016 y junio de 2017 y se han obtenido de diferentes plataformas, algunas de ellas utilizadas en diferentes sectores (como LinkedIn ${ }^{11}$ o portales como Glassdoor ${ }^{12}$ o Infoempleo ${ }^{13}$ ) y otras propias del sector de la traducción (Europe Language Jobs ${ }^{14}$, GALA ${ }^{15}$, Lion People ${ }^{16}$, The Translation People ${ }^{17}$ ). Además, también se han encontrado ofertas en páginas de empresas pertenecientes a diferentes sectores que precisan de un gestor de proyectos de traducción (Amazon ${ }^{18}$, Facebook $^{19}$, VMware ${ }^{20}$, por mencionar algunos ejemplos) o de grandes empresas de traducción (Amplexor ${ }^{21}$, Lionbridge ${ }^{22}, \mathrm{SDL}^{23}$, Smartling ${ }^{24}$, Transperfect ${ }^{25}$, etc.). Con esta variedad de plataformas se pretende evitar el uso repetido de modelos establecidos por los sitios web para la descripción de tareas y competencias, pero se ha tenido especial cuidado para que los anuncios no estuvieran duplicados.

Durante la fase de búsqueda y recopilación de los anuncios, aquellos que eran seleccionados se archivaban guardando una copia impresa en PDF, donde se incluía también la dirección URL de la que se había extraído, para evitar perderlo si era retirado del sitio web. Entre los criterios de selección, cabe destacar que la mayor parte de los anuncios solicitaban un project manager. No se incluyeron figuras como vendor manager, account manager o program manager, salvo en contadas ocasiones en las que tras analizar el contenido se podía concluir que las funciones y las competencias 
descritas pertenecían al PM y coincidían en parte con el resto de anuncios analizados. Asimismo, durante esta fase se eliminaron 58 de los 158 anuncios recopilados inicialmente, bien por estar repetidos, incluir una descripción pobre o incompleta, haber desaparecido o no estar dirigidos a gestores de proyectos de traducción.

En lo que respecta a la metodología empleada, aunque en un principio se planteó la opción de analizarlo mediante una herramienta de análisis de corpus, como WordSmith Tools, tras los primeros análisis se detectó que la formulación de las tareas y de las competencias descritas en los anuncios variaba entre unos y otros. De esta forma, se podrían obviar inintencionadamente algunas competencias y no analizar los anuncios en su totalidad. Por este motivo, se optó por el análisis de contenido como técnica de investigación, ya que de esta forma los datos son filtrados por el investigador y se organizan haciendo uso de categorías (López Noguero 2002: 169; Ahsan, Ho, et al. 2013: 43). Para evitar problemas de subjetividad e interpretación incorrecta de datos dada la cantidad de anuncios, se realizaron dos análisis separados en el tiempo que permitieron corroborar la información y resolver errores.

Para organizar el contenido analizado, se identificaron una serie indicadores textuales que hacían referencia a tareas y competencias recurrentes. Se tomaron como referencia los dos modelos de competencias mencionados anteriormente, el de PACTE y el PMCD Framework y, partiendo de estos modelos, se catalogaron los distintos componentes mencionados en los anuncios según las categorías establecidas: subcompetencia bilingüe, extralingüística, de conocimientos de traducción, instrumental, estratégica y componentes psicofisiológicos. En estas dos últimas categorías se tuvieron en cuenta las dimensiones de desempeño y personal descritas por el $P M C D$ Framework.

Como se ha adelantado, del corpus de anuncios no solo se analizaron las competencias bajo epígrafes como «Competencias deseadas», sino que también se tuvieron en cuenta las descripciones de las tareas que debe realizar el gestor de proyectos. Se tomó esta decisión porque en algunos anuncios no se buscan a profesionales con amplia formación o experiencia previa en la gestión de proyectos, por lo que en la descripción de competencias simplemente se establecen una serie de competencias básicas sobre las que desarrollar otras propias del gestor de proyectos. Así pues, para extraer conclusiones, ha sido necesario considerar las tareas descritas y traducir dicho lenguaje a competencias. Así, por ejemplo, expresiones como cumplir la fecha de entrega de los clientes, se han incluido en la capacidad de trabajar bajo presión, y las tareas de elaboración de informes sobre el rendimiento del equipo se engloban en la categoría de habilidad de análisis.

En definitiva y en consonancia con Ahsan, Ho, et al. (2013), el enfoque de análisis de contenido adoptado ha permitido realizar inferencias del texto y obtener datos, por un lado, de carácter cualitativo, que proporcionen un marco conceptual, y por otro, cuantitativo, que nos permita resumir las inferencias derivadas del análisis cualitativo en forma de datos numéricos.

Se debe puntualizar que el corpus cuenta con una serie de limitaciones que se deben tener en cuenta a la hora de interpretar los datos. En primer lugar, hay que señalar que se trata de una muestra pequeña si tenemos en cuenta que intenta representar el mercado de trabajo a escala mundial. Además, se ha llegado a la conclusión de que las denominaciones empleadas para designar cada puesto de trabajo varían mucho de una empresa a otra y, en ocasiones, la experiencia requerida también es 
diferente. Así pues, es posible encontrar perfiles de gestores de proyectos más centrados en la gestión de equipo y de la cartera de clientes, mientras que otros ponen el acento en tareas de calidad y gestión del material. Por este motivo, se ha intentado que todos estos perfiles y tareas tengan representación en la muestra. Por último, es conveniente señalar que la redacción de un anuncio puede ser muy variada y, en ocasiones, no se nombran determinadas subcompetencias, sin que ello quiera decir que no se valoren a la hora de conseguir el puesto de trabajo.

\subsection{Análisis del corpus}

En primer lugar, resumiremos una serie de datos de carácter cualitativo sobre los anuncios que constituyen el corpus: naturaleza de las empresas demandantes, denominación del puesto, experiencia previa necesaria y nivel de estudios o titulación.

Las empresas cuyos anuncios forman parte del corpus se pueden categorizar de la siguiente forma: de los 100 anuncios, 55 empresas eran de traducción, 15 empresas del ámbito tecnológico, 3 empresas multimedia, 1 de formación en línea y 1 de comercio electrónico. El resto de anuncios no ofrece ninguna información al respecto.

La denominación más utilizada para nombrar el puesto de trabajo es la de project manager, con un total de 84 anuncios, aunque esta cifra puede estar sesgada, ya que en aquellas plataformas que no permitían buscar por categorías, se introdujo este término de búsqueda. Suelen distinguir entre localization o translation project manager, por lo que sería interesante investigar si existen diferencias notables entre ambos perfiles. Igualmente mencionan las lenguas de trabajo, la especialidad en la que se va a trabajar o la categoría (júnior, sénior, assistant, etc.). En 9 de los anuncios se habla de project coordinator y en un solo caso se denomina project leader. Aunque la $P_{M B O K^{\oplus}}$ Guide distingue entre program management, operations management y project management, en nuestro corpus se han incluido 3 anuncios que buscan un localization program manager y otro en el que se adopta la denominación de language services operations associate. Las funciones y competencias descritas coincidían en gran parte con el resto de anuncios, por lo que se ha dado prioridad a la función frente al título. Mención especial merecen las denominaciones de senior translation account manager y marketing localization producer. La primera se ha incluido porque una cuenta normalmente hace referencia a un conjunto de proyectos de un cliente que comparten ciertas características en común (producto, departamento al que pertenece, tareas que se realizan), por lo cual la función descrita es la misma que para un PM pero aplicada a una sola cuenta. El segundo caso es una nomenclatura que adopta la empresa, a pesar de que en el mismo anuncio se especifica «[w]e're looking for a highly dedicated and experienced project manager $»^{26}$.

En lo que se refiere a la experiencia deseada para acceder al puesto, en la mayoría de los casos se indica un intervalo de tiempo, por ejemplo, de 1 a 3 años. Para resumir los datos, se han agrupado según la experiencia mínima requerida para poder optar al puesto de trabajo, ya que es la cifra a partir de la cual se consideraría válida una candidatura. 
CUADRO 2

Experiencia previa necesaria

\begin{tabular}{|l|c|}
\hline N. ${ }^{\circ}$ de años de experiencia & N.o de anuncios \\
\hline No especificado & 34 \\
\hline 0 años & 1 \\
\hline 1 año o más & 13 \\
\hline 2 años o más & 19 \\
\hline 3 años o más & 14 \\
\hline 4 años o más & 4 \\
\hline 5 años o más & 15 \\
\hline
\end{tabular}

Por último, en lo que concierne a la titulación o el nivel de estudios deseado, en ocasiones se nombran varias titulaciones, por ejemplo, graduado en Traducción o Lenguas modernas, o graduado en Ingeniería o Localización.

CUADRO 3

Titulación necesaria

\begin{tabular}{|l|c|}
\hline Titulación & N. ${ }^{\circ}$ de anuncios \\
\hline No especificada & 28 \\
\hline Graduado & 24 \\
\hline Traducción o similar & 25 \\
\hline Empresariales & 13 \\
\hline Ingeniería o similar & 12 \\
\hline Gestión de proyectos & 1 \\
\hline
\end{tabular}

Aunque el grado en Traducción (o similar) es el más mencionado con un 25\%, los anuncios que demandan Empresariales o Ingeniería suman la misma cantidad que los primeros. Asimismo, llama la atención que solo un caso menciona una titulación en Gestión de proyectos. De estos datos se podrían extraer tres conclusiones: que falte información en los anuncios sobre la titulación; que los empleadores consideren que son necesarias competencias de otras titulaciones y que, con una breve formación en aspectos relativos a la gestión de proyectos de traducción, cualquier titulado puede desempeñar estas funciones, o que no otorguen importancia a los conocimientos específicos en traducción. Sin embargo, como señala el PMI cuando indica que es necesario desarrollar modelos específicos de competencias para cada sector (Ahsan, Ho, et al. 2013: 43) y como demostrarán los datos que a continuación se exponen, todo gestor de proyectos debería poseer conocimientos propios del campo en el que lleva a cabo sus funciones. Es imposible realizar una estimación de la duración o de los costes de un proyecto de localización, por ejemplo, si antes no se han adquirido conocimientos sobre las variables que influyen en dicho cálculo. Asimismo, la gestión de riesgos o de recursos humanos solo es viable si se conoce el proceso de traducción en su totalidad.

Antes de empezar a desglosar los datos por subcompetencias, resulta interesante enumerar los componentes más importantes según el corpus. Para hacer referencia a las habilidades/capacidades mencionadas en los anuncios, se utilizará de manera global el término componentes de las subcompetencias, sin diferenciar entre unidades o elementos como ocurre en el PMCD Framework. 
CUADRO 4

Componentes más importantes de las competencias del gestor de proyectos

\begin{tabular}{|l|c|}
\hline Componente de la subcompetencia & Porcentaje \\
\hline Trabajo en equipo & $98 \%$ \\
\hline Trato con cliente o proveedores & $95 \%$ \\
\hline Habilidades comunicativas & $91 \%$ \\
\hline Gestión del tiempo & $89 \%$ \\
\hline Conocimientos informáticos avanzados & $77 \%$ \\
\hline Dominio de lengua extranjera & $74 \%$ \\
\hline Realización de tareas administrativas & $66 \%$ \\
\hline Capacidad de trabajar bajo presión & $64 \%$ \\
\hline Dominio del inglés & $63 \%$ \\
\hline Solución de problemas & $63 \%$ \\
\hline
\end{tabular}

Como reflejan estos resultados, las habilidades relacionadas con el trabajo en equipo y con cliente y proveedores, categorizadas bajo la subcompetencia estratégica, ocupan los dos primeros puestos, ya que en la gestión de proyectos resulta esencial la colaboración entre las distintas partes implicadas en el proceso. Las habilidades comunicativas, en tercer lugar, no hacen referencia tanto al uso de una lengua en concreto, sino más bien a la capacidad de comunicarse con el equipo de manera eficaz, intercambiar de forma adecuada y precisa la información con las partes implicadas y adaptar el mensaje a los receptores utilizando los métodos adecuados (PMI 2002/2007: 26). Por consiguiente, se confirma que el PM actúa de eslabón comunicativo entre los participantes en el proceso. No obstante, el dominio de otras lenguas también se encuentra entre los primeros diez puestos, por lo que la subcompetencia bilingüe del traductor adquiere gran relevancia. Esta lista de los diez componentes más importantes de las competencias del PM supone una representación de prácticamente todas las subcompetencias mencionadas por PACTE (a excepción de la subcompetencia extralingüística), lo cual pone de relieve la necesidad del PM de estar familiarizado con la traducción y viene a rebatir los datos arrojados anteriormente sobre la titulación exigida en los anuncios.

A continuación, para facilitar la exposición de los resultados, estos se organizarán según las distintas subcompetencias mencionadas por PACTE. En relación con la primera de ellas, la subcompetencia bilingüe, encontramos los siguientes componentes:

CuAdRO 5

Componentes de la subcompetencia bilingüe

\begin{tabular}{|l|c|}
\hline Componente de la subcompetencia & Porcentaje \\
\hline Dominio de lengua extranjera & $74 \%$ \\
\hline Dominio del inglés & $63 \%$ \\
\hline Control de calidad lingüística & $19 \%$ \\
\hline Traducción & $8 \%$ \\
\hline
\end{tabular}

Cabe señalar que en ocasiones no queda claro si el dominio de una o varias lenguas extranjeras incluye o excluye al inglés. Por ello, durante el segundo análisis 
del corpus, se consideró importante separarlos, ya que el inglés se utiliza como lengua vehicular y en muchos anuncios se vincula con las habilidades comunicativas con expresiones como: «[e]xcellent written and verbal English communication skills» ${ }^{27} \mathrm{o}$ "[f]luency in English which is our corporate language» ${ }^{28}$. Asimismo, hay que añadir que el dominio de una lengua extranjera (que no sea inglés) se suele incluir en muchos anuncios como una capacidad deseada, aunque no imprescindible.

Los otros dos componentes localizados en los anuncios y relacionados con la subcompetencia bilingüe podrían ser más propios del perfil de un traductor. Por un lado, se encuentra el control de calidad lingüística. Aunque no es un porcentaje alto, llama la atención que en el 19\% de los casos esta función recaiga al menos en parte en dicha figura, dada la gran cantidad de tareas que desempeña. Nótese que aquí no se incluyen las menciones a la gestión propiamente dicha de la calidad del proyecto, entendido como proceso, ya que se incluyen en el componente de análisis y gestión de la calidad, los costes y los plazos de la subcompetencia estratégica. Por último, en un $8 \%$ de los anuncios se especifica que el gestor de proyectos debe ser capaz de realizar traducciones. No se precisa un volumen diario y ninguno de los anuncios que menciona este componente coincide con perfiles complejos de gestión de proyectos que impliquen tareas comerciales o de elaboración de informes que les impidan realizar tareas productivas.

La subcompetencia extralingüística podría considerarse la gran olvidada en el perfil del PM según nuestro corpus. Aunque se puede deducir que el conocimiento de una lengua extranjera implica también conocimientos culturales y que la realización de traducciones supone determinados conocimientos enciclopédicos, en ningún anuncio se hace referencia explícita a ellos. Solo en un caso se señala que son necesarios conocimientos en música, un campo poco común. No obstante, en 10 anuncios se nombra una especialidad en la denominación del puesto (jurídica, financiera, médica, etc.) y solo en 3 más se concreta la naturaleza de los proyectos que se van a gestionar. Estos datos podrían indicar que en el caso de que un PM gestione proyectos especializados en un campo, se podrían requerir conocimientos específicos en la materia. Es necesario profundizar en esta cuestión en futuros trabajos, pero con los datos del presente estudio se puede intuir que, al igual que para gestionar proyectos de traducción se deberían poseer conocimientos en este campo, para la gestión de proyectos especializados en distintas áreas de la traducción, se podrían precisar de conocimientos específicos para una correcta ejecución del proyecto.

La categorización de los componentes de las subcompetencias puede resultar una tarea compleja, ya que estas se encuentran interrelacionadas y en ocasiones los límites se solapan. Tal es el caso de la subcompetencia de conocimientos de traducción, en la que se podría incluir, por ejemplo, la realización de controles de calidad lingüística, ya que implica conocimientos sobre los tipos de encargo, el público objetivo, etc. Lo mismo ocurre con la habilidad de análisis, puesto que, para poder llevar a cabo tareas de índole analítica, se requieren conocimientos declarativos sobre la profesión. Por ello, con el fin de evitar la duplicidad en el análisis, en aquellos casos en los que era posible categorizar el componente en dos subcompetencias diferentes, se optó por aquella que se puede considerar predominante en el componente en cuestión. Así pues, para la subcompetencia de conocimientos de traducción se pueden detallar los siguientes componentes: 
CUADRO 6

Componentes de la subcompetencia de conocimientos de traducción

\begin{tabular}{|l|c|}
\hline Componente de la subcompetencia & Porcentaje \\
\hline Realización de tareas administrativas & $66 \%$ \\
\hline Habilidades comerciales & $36 \%$ \\
\hline Preparación de material de referencia & $26 \%$ \\
\hline Comprensión de instrucciones & $9 \%$ \\
\hline
\end{tabular}

El primero de los componentes incluye la emisión de órdenes de pedido (PO, purchase order), facturas, realización y control de presupuestos, supervisión de la rentabilidad de un proyecto e incluso preparación de propuestas de trabajo para potenciales clientes, tarea que roza el límite con las habilidades comerciales, el segundo de los componentes mencionado en el cuadro. Tal es la importancia de esta subcompetencia que, como se refleja en el Cuadro 4, ocupa el octavo lugar entre las subcompetencias más importantes de este perfil según el corpus.

En lo que concierne a las habilidades comerciales, entre ellas se incluyen tareas de búsqueda y establecimiento de relaciones con nuevos clientes, planificación de reuniones o negociación de las condiciones del contrato, como las tarifas, ya sea con clientes o proveedores. Se encuentran íntimamente relacionadas con la subcompetencia estratégica. El porcentaje para este componente es de un $36 \%$, una cifra poco elevada, pero al mismo tiempo justificada, dada la variedad de perfiles de gestión de proyectos detectados en el corpus.

Los dos últimos componentes cobran menos relevancia en lo que a porcentajes se refiere. Ambos pueden tener repercusión directa en el proceso de traducción o revisión, dado que una mala preparación del material y una incorrecta comprensión de instrucciones pueden provocar pérdidas en el proyecto. Sin embargo, según el corpus, no parecen constituir subcompetencias esenciales para el PM, quizás porque son tareas que se delegan en parte a otros profesionales de la cadena productiva.

Los datos obtenidos para la subcompetencia instrumental son los siguientes:

\section{CUADRO 7}

Componentes de la subcompetencia instrumental

\begin{tabular}{|l|c|}
\hline Componente de la subcompetencia & Porcentaje \\
\hline Conocimientos informáticos avanzados & $77 \%$ \\
\hline Preparación y gestión de archivos & $23 \%$ \\
\hline Conocimientos informáticos básicos & $12 \%$ \\
\hline Habilidades documentales & $3 \%$ \\
\hline
\end{tabular}

La distinción entre conocimientos informáticos básicos y avanzados radica en que en el segundo caso se requiere el uso de herramientas propias del sector, como herramientas de traducción asistida por ordenador (TAO), o conocimientos informáticos avanzados en suites de ofimática u otras herramientas propias de la gestión. En cambio, en aquellos anuncios categorizados bajo la etiqueta de conocimientos informáticos básicos, simplemente se requiere un nivel de conocimientos intermedio de herramientas básicas o, como se indica en varios casos, «afinidad» con las nuevas tecnologías y ganas de aprender. Como revela el cuadro, únicamente en un $12 \%$ se 
requieren conocimientos básicos. Es necesario destacar que solo en un $10 \%$ de los anuncios se menciona el manejo de herramientas de gestión de proyectos como requisito deseado del candidato, por lo que se puede concluir que en este corpus se concede más importancia al manejo de herramientas TAO que a herramientas propias de gestión.

Un porcentaje similar a la preparación de archivos de referencia, mencionado en la subcompetencia anterior, recibe la preparación y gestión de los archivos de traducción, con solo un $23 \%$. Al igual que ocurría con los archivos de referencia, se deduce que esta tarea la pueden realizar otros miembros del equipo. Y, por último, debemos destacar el bajo porcentaje que obtienen las habilidades documentales o de investigación, que solo se mencionan en 3 de los 100 anuncios. Al igual que ocurría con la subcompetencia extralingüística, según los datos aquí extraídos, estas dos subcompetencias parecen más propias de la figura del traductor.

En lo que respecta a la subcompetencia estratégica, gracias al corpus se han detectado los siguientes componentes:

\section{CUADRO 8}

\section{Componentes de la subcompetencia estratégica}

\begin{tabular}{|l|c|}
\hline Componente de la subcompetencia & Porcentaje \\
\hline Trabajo en equipo & $98 \%$ \\
\hline Trato con cliente o proveedores & $95 \%$ \\
\hline Gestión del tiempo & $89 \%$ \\
\hline Solución de problemas & $63 \%$ \\
\hline Coordinación del proyecto de principio a fin & $57 \%$ \\
\hline Multitarea & $47 \%$ \\
\hline Habilidad de análisis & $33 \%$ \\
\hline Análisis y gestión de calidad, costes y plazos & $25 \%$ \\
\hline
\end{tabular}

De los 8 componentes del cuadro, los 4 primeros se encuentran entre las 10 habilidades más importantes del gestor de proyectos, según el cuadro 4 . Al igual que ocurría en otras subcompetencias, con algunos de estos componentes podría existir confusión a la hora de categorizarlos, ya que la multitarea o el trabajo en equipo, por ejemplo, requieren de determinadas habilidades cognitivas propias de los componentes psicofisiológicos. No obstante, dada su relación con el proceso de traducción y el mayor peso de los componentes estratégicos, se ha preferido optar por su inclusión en esta subcategoría.

Los dos primeros componentes se encuentran íntimamente relacionados, ya que el trato con clientes o proveedores también implica en cierto modo trabajo en equipo, aunque no suponga la movilización de las mismas habilidades. En un principio, se consideró la opción de distinguir el trabajo en equipo, como trabajo entre pares, de la gestión o supervisión de equipo, pero finalmente se consideró que esta última es parte fundamental de la primera, ya que independientemente del cargo, ambas tareas suponen trabajar mano a mano. Se ha de destacar el fuerte componente psicofisiológico del trabajo en equipo; su inclusión en esta categoría viene justificada por el hecho de que la gestión de los recursos humanos es parte fundamental del proceso de planificación y ejecución de un proyecto, pero esto no implica que sin las habilidades psicofisiológicas necesarias se consiga una buena gestión del equipo. Los porcentajes 
tanto de la capacidad de trabajar en equipo como de tratar con clientes o proveedores (98\% y 95\% respectivamente) y su posicionamiento como los dos componentes más importantes de las competencias del gestor de proyectos, según este estudio, evidencian el papel del PM en la comunicación y supervisión durante el ciclo de vida del proyecto.

La gestión del tiempo se convierte en el tercer componente de mayor importancia dentro de esta categoría. El tiempo constituye una de las tres variables troncales (Matcha Abombo 2015: 151) de la gestión de proyectos, junto con la calidad y los costes, y se puede entender de dos formas: gestión de los plazos establecidos por los clientes y gestión del tiempo del equipo (traductores, revisores, maquetadores, etc.). Por el elevado porcentaje de aparición en el corpus de anuncios, es posible afirmar que el factor tiempo desempeña un papel crucial en el análisis de las tres variables troncales, aunque como demuestran los datos el control de los costes y de la calidad del proyecto también tienen un gran peso. En referencia a dichas variables, se encuentra el componente que en este estudio hemos denominado "Análisis y gestión de calidad, costes y plazos» y que se menciona en el 25\% de los anuncios. Aunque no supone un porcentaje elevado, en el segundo análisis realizado se decidió separar de las tareas puramente administrativas (como emisión de presupuestos o PO) por incluir el factor calidad y tiempo y estar presente en diferentes anuncios con expresiones como «show good judgment in balancing budget, quality, timelines, and deliverables $»^{29} \mathrm{O}$ «[m] anage quality, costs and schedules for your projects $»^{30}$.

El componente de solución de problemas, con una representación del 63\% en el corpus, ha conseguido abrirse hueco también entre las 10 habilidades más importantes del gestor de proyectos de traducción. En esta categoría se han incluido aquellos anuncios que nombran tanto la identificación y solución de problemas como la toma de decisiones. Igualmente, la identificación de riesgos y una planificación estratégica para minimizarlos se ha contabilizado en un $21 \%$ de los anuncios. La gestión de riesgos constituye de por sí un área de conocimiento de la $P M B O K^{\oplus}$ Guide y ha recibido especial atención en tesis doctorales como la de Dunne (2013).

El siguiente componente, la coordinación de proyectos de principio a fin (57\%), coincide íntegramente con la dimensión de desempeño del PMCD Framework. Las 5 unidades que se diferencian en dicho trabajo para la dimensión de desempeño son:

- inicio del proyecto;

- planificación del proyecto;

- ejecución del proyecto;

- supervisión y control del proyecto;

- cierre del proyecto.

Aunque en parte podría incluirse dentro de la subcompetencia de conocimientos de traducción, predominan los conocimientos procedimentales y no tanto declarativos. Además, podría considerarse como una macrocompetencia que abarca a todas las demás y donde entran en juego la mayoría de ellas: gestión de equipo, gestión del tiempo, análisis de las tres variables troncales, preparación y gestión de archivos, etc.

Otra de las habilidades que suele caracterizar a un gestor de proyectos es la multitarea, no solo gestionando proyectos diferentes, sino también realizando informes, gestionando recursos, supervisando a un equipo, entre otras. Por ese motivo, este componente tiene una representación del $47 \%$ en el corpus y en el $31 \%$ se habla de saber priorizar a la hora de hacer frente a diferentes tareas. 
En habilidad de análisis (33\%) se han incluido aquellos anuncios que mencionan directamente las habilidades analíticas para gestionar un proyecto y aquellos que incluyen la redacción de informes de diversa naturaleza: análisis de procesos, key performance indicators (KPI), rendimiento del equipo, rentabilidad de proyectos, etc. Como indica el porcentaje obtenido, la realización de informes no es una tarea exigida para la mayoría de los puestos, pero sí se ha observado cierta tendencia a incluirse en anuncios cuya descripción está más relacionada con la gestión y supervisión a niveles macroestructurales y no tanto de proyectos.

Los componentes psicofisiológicos, aunque no constituyan una subcompetencia en sí, han recibido bastante atención desde la investigación en gestión de proyectos y recursos humanos y en ocasiones se les ha denominado «soft skills» $\mathrm{u}$ «other attributes» (Ahsan, Ho, et al. 2013: 40).

CUADRO 9

Componentes psicofisiológicos

\begin{tabular}{|l|c|}
\hline Componente & Porcentaje \\
\hline Habilidades comunicativas & $91 \%$ \\
\hline Capacidad de trabajar bajo presión & $64 \%$ \\
\hline Capacidad organizativa & $58 \%$ \\
\hline Atención al detalle & $46 \%$ \\
\hline Motivación & $39 \%$ \\
\hline Autonomía & $39 \%$ \\
\hline Habilidades interpersonales & $34 \%$ \\
\hline Actitud proactiva & $23 \%$ \\
\hline Actitud positiva & $22 \%$ \\
\hline Capacidad de adaptarse a diferentes situaciones & $14 \%$ \\
\hline Liderazgo & $11 \%$ \\
\hline Responsabilidad & $5 \%$ \\
\hline
\end{tabular}

En este cuadro, se puede observar que es la categoría que más componentes incluye y teniendo en cuenta las peculiaridades de los anuncios, con un corpus de mayor tamaño posiblemente se hubieran extraído otros que se mencionan en el PMCD Framework, como la profesionalidad o la eficacia.

Las habilidades comunicativas y la capacidad de trabajar bajo presión (91\% y $64 \%$ respectivamente) se encuentran entre las habilidades más importantes para el gestor de proyectos. Aunque de manera tangencial, ya se ha comentado la relevancia de ambos. El primero de ellos implica, como indica el PMCD Framework (PMI 2002/2007: 25), escuchar, comprender y responder de manera activa a las partes implicadas, mantener las líneas de comunicación, garantizar la calidad de la información y adaptar el mensaje al destinatario. La capacidad de trabajar bajo presión se encuentra directamente vinculada con la multitarea, la gestión del tiempo y las capacidades organizativas del PM, que como demuestra el cuadro 9, obtiene un 58\% de representación, justo por debajo de la capacidad de trabajar bajo presión.

La atención al detalle, con un $46 \%$, hace referencia a la capacidad del gestor de proyectos de estar pendiente de los diferentes elementos y factores que confluyen en el proceso. Ha llamado la atención especialmente que es una competencia que en los 
anuncios se menciona tal cual, «attention to detail», y no ha sido necesario definir indicadores textuales que pudieran hacer referencia a dicha capacidad.

Bajo la categoría de componentes actitudinales se podrían incluir aspectos como la motivación, la autonomía, una actitud positiva y proactiva y la responsabilidad. Aunque no son porcentajes muy elevados, la suma de todos ellos supone una gran representación en el corpus y, como se ha mencionado, a esta lista sería posible añadir otros muchos con un corpus mayor. Asimismo, se encuentran en cierto modo vinculados, ya que por ejemplo una actitud proactiva implica cierta autonomía y una actitud positiva conlleva también motivación.

Las habilidades interpersonales también cuentan con una representación del 34\%. Aunque en el PMCD Framework no se menciona ninguna unidad con dicho nombre, su presencia se hace patente en cada uno de sus elementos con expresiones como adaptar la comunicación a la audiencia, motivar a los miembros del equipo o emplear la asertividad cuando sea necesario.

La capacidad de adaptarse a diferentes situaciones toma diferentes formas en el texto de los anuncios, con expresiones del tipo "una persona flexible, receptiva» o «capacidad de aplicar cambios eficazmente». Aunque su nivel de representación en el corpus es bajo, este componente se encuentra implícito en la capacidad de multitarea del PM. Por último, llama la atención que la capacidad de liderazgo solo se mencione en un $11 \%$ de los anuncios, aunque se podría concluir que de la gestión y supervisión de equipo se deduce cierta capacidad de liderazgo. El PMCD Framework sí lo incluye como una unidad dentro de las competencias personales.

\section{Conclusiones}

Sin olvidar las limitaciones mencionadas en el apartado de diseño del corpus y metodología de análisis, el presente estudio es un primer paso en la definición de las competencias del gestor de proyectos de traducción. A pesar de ser una muestra relativamente pequeña y de corroborar la variedad de perfiles existentes dentro de la gestión de proyectos, los datos extraídos ofrecen una amplia perspectiva de las demandas de los empleadores. En el siguiente cuadro se resumen los principales componentes de las subcompetencias del gestor de proyectos de traducción:

\section{CUADRO 10}

\section{Competencias del gestor de proyectos de traducción}

\begin{tabular}{|c|c|c|}
\hline Subcompetencia bilingüe & $\begin{array}{l}\text { Subcompetencia de conocimientos de } \\
\text { traducción }\end{array}$ & $\begin{array}{l}\text { Subcompetencia } \\
\text { instrumental }\end{array}$ \\
\hline Dominio de lengua extranjera & Realización de tareas administrativas & $\begin{array}{l}\text { Conocimientos informáticos } \\
\text { avanzados }\end{array}$ \\
\hline Dominio del inglés & Habilidades comerciales & $\begin{array}{l}\text { Preparación y gestión de } \\
\text { archivos }\end{array}$ \\
\hline Control de calidad lingüística & Preparación de material de referencia & $\begin{array}{l}\text { Conocimientos informáticos } \\
\text { básicos }\end{array}$ \\
\hline Traducción & Comprensión de instrucciones & Habilidades documentales \\
\hline
\end{tabular}




\begin{tabular}{|l|l|}
\hline Subcompetencia estratégica & Componentes psicofisiológicos \\
\hline Trabajo en equipo & Habilidades comunicativas \\
Trato con cliente o proveedores & Capacidad de trabajar bajo presión \\
Gestión del tiempo & Capacidad organizativa \\
Solución de problemas & Atención al detalle \\
Coordinación del proyecto de principio a fin & Motivación \\
Multitarea & Autonomía \\
Habilidad de análisis & Habilidades interpersonales \\
Análisis y gestión de calidad, costes y plazos & Actitud proactiva \\
& Actitud positiva \\
& Capacidad de adaptarse a diferentes situaciones \\
& Liderazgo \\
& Responsabilidad \\
\hline
\end{tabular}

Como se puede observar, ha sido posible acotar las principales competencias de los PM a partir de aquellas definidas para los traductores. Esto permite concluir que la formación de ambos perfiles debe partir de la misma base, aunque primarán unos componentes sobre otros en función de las tareas realizadas. Así pues, mientras que para el traductor resulta fundamental la subcompetencia bilingüe e incluso la extralingüística (subcompetencia que en nuestro análisis apenas cuenta con representación), para el gestor de proyectos la subcompetencia estratégica desempeña un papel crucial. La subcompetencia bilingüe también ocupa un lugar relevante en los anuncios del corpus, pero prevalecen las habilidades comunicativas, uno de los componentes psicofisiológicos más mencionados. En lo que concierne a la subcompetencia instrumental, sí se observa cierta similitud entre los conocimientos exigidos al traductor y al PM, coincidiendo así con los resultados del estudio realizado por Bowker (2004: 971), limitado al mercado canadiense, aunque perfectamente extrapolable a otras partes del mundo. La única diferencia notable podría ser la capacidad de preparación y gestión de archivos, aunque no ha demostrado tener una elevada representación en el corpus. Por su parte, en lo que respecta a la subcompetencia de conocimientos de traducción, pese a que no se hace referencia a conocimientos sobre el funcionamiento de la traducción (métodos, procedimientos, estrategias o técnicas), sí adquieren gran importancia los conocimientos relacionados con la profesión. Este dato corrobora la necesidad de contar con gestores con conocimientos en traducción, a pesar de que en los anuncios el nivel de estudios o titulación no siempre hiciera referencia a esta formación. En relación con los componentes psicofisiológicos, se puede afirmar que la mayoría de ellos son perfectamente aplicables al perfil del traductor.

A pesar de la dificultad que implica el análisis de contenidos, por no ser una técnica de análisis automático de información, los resultados obtenidos en este trabajo son un primer intento de acotar las competencias del gestor de proyectos en traducción. Es posible confirmar que las áreas de conocimiento descritas en la $P M B O K^{\oplus}$ Guide (PMI 1996/2013) y las competencias definidas en el PMCD Framework (PMI 2002/2007) son aplicables al sector de la traducción, al mismo tiempo que se pueden integrar con modelos específicos de competencias del traductor. No obstante, se precisan de investigaciones que se centren en las diferentes áreas de conocimiento de la $P M B O K^{\oplus}$ Guide (PMI 1996/2013) aplicadas a la traducción y la interpretación, en 
la definición de las competencias por parte de los formadores, así como en los diferentes perfiles de PM o en las competencias específicas que se han puesto de manifiesto con este estudio. Todas ellas son futuras líneas de investigación que ya contempla la autora de este artículo.

En definitiva, en un esfuerzo por alinear las competencias definidas para la educación y el mercado laboral, este trabajo pretende poner de relieve que, a pesar de la idiosincrasia de las empresas, es posible partir de una base sobre la que empezar a construir y desarrollar competencias para la educación, que posteriormente se adaptarán a necesidades empresariales específicas. Este estudio se podría complementar, además, con otras técnicas de obtención de datos, como encuestas o entrevistas o la observación directa de tareas.

\section{NOTES}

1. En las referencias bibliográficas se menciona el nombre completo del instituto, Project Management Institute.

2. Asociación Española de Normalización y Certificación (2015): UNE-EN ISO 17100: Servicios de traducción. Requisitos para los servicios de traducción. Consultado el 21 de agosto de 2017, <https://www.une.org/ encuentra-tu-norma/busca-tu-norma/norma/?c=N0055778>.

3. Project Management Institute (Actualizado el 19 de julio de 2017): Consultado el 24 de julio de 2017, <https://www.pmi.org/>.

4. International Project Management Association (Actualizado el 11 de julio de 2017): Consultado el 24 de julio de 2017, <http://www.ipma.world/>.

5. Association for Project Management (Actualizado el 21 de julio de 2017): Consultado el 24 de julio de 2017, <https://www.apm.org.uk/>.

6. La tercera versión del PMCD Framework se publicó en marzo de 2017, pero no ha sido posible acceder a ella.

7. Torres-Simón, Ester y Pym, Anthony (2017): European Masters in Translation. A Comparative Study. Consultado el 21 de agosto de 2017, <https://www.researchgate.net/ publication/314090217_ European_Masters_in_Translation_A_comparative_study $>$.

8. Stoeller, Willem (2004): The hub of the wheel. MultiLingual Computing \& Technology. Supplement 63:3-6. Consultado el 28 de agosto de 2017, <https://multilingual.com/downloads/ screenSupp63.pdf>.

9. PArrish, Donna, ed. (2004): The Guide to Project Management. MultiLingual Computing \& Technology. Supplement 63. Consultado el 28 de agosto de 2017, <https://multilingual.com/downloads/screenSupp63.pdf $>$.

10. Toudic, Daniel y Krause, Alexandra (de parte del EMT Board) (2017): Competence Framework 2017. European Master's in Translation. Consultado el 9 de julio de 2018, <https://ec.europa.eu/ info/sites/info/files/emt_competence_fwk_2017_en_web.pdf>.

11. LinkedIn (Actualizado el 27 de agosto de 2017): Consultado el 28 de agosto de 2017, <https://www. linkedin.com/>.

12. Glassdoor (Actualizado el 28 de agosto de 2017): Consultado el 28 de agosto de 2017, <https://www. glassdoor.com/index.htm>.

13. Infoempleo (Actualizado el 22 de agosto de 2017): Consultado el 28 de agosto de 2017, <https:// www.infoempleo.com/>.

14. Europe Language Jobs (Actualizado el 22 de julio de 2017): Consultado el 28 de agosto de 2017, $<$ https://www.europelanguagejobs.com/>.

15. Globalization \& Localization Association (Actualizado el 10 de agosto de 2017): Consultado el 28 de agosto de 2017, <https://www.gala-global.org/>.

16. Lion People (Actualizado el 9 de julio de 2017): Consultado el 28 de agosto de 2017, <https://lionpeopleglobal.com/>.

17. The Translation People (Actualizado el 20 de agosto de 2017): Consultado el 28 de agosto de 2017, <http://www.thetranslationpeople.com/>.

18. Amazon (Actualizado el 27 de agosto de 2017): Consultado el 28 de agosto de 2017, <https://www. amazon.com/>. 
19. Facebook (Actualizado el 27 de agosto de 2017): Consultado el 28 de agosto de 2017, <https://www. facebook.com/>.

20. VMWare (Actualizado el 26 de agosto de 2017): Consultado el 28 de agosto de 2017, <https://www. vmware.com/>.

21. Amplexor (Actualizado el 20 de agosto de 2017): Consultado el 28 de agosto de 2017, <http://www. amplexor.com/>.

22. Lionbridge (Actualizado el 25 de agosto de 2017): Consultado el 28 de agosto de 2017, <https:// www.lionbridge.com>.

23. SDL (Actualizado el 24 de agosto de 2017): Consultado el 28 de agosto de 2017, <http://www.sdl. com>.

24. Smartling (Actualizado el 22 de agosto de 2017): Consultado el 28 de agosto de 2017, <https://www. smartling.com/>.

25. Transperfect (Actualizado el 13 de junio de 2017): Consultado el 28 de agosto de 2017, <http:// es.transperfect.com/>.

26. Apple (2017): Marketing Localization Producer. Apple. Consultado el 24 de junio de 2017, <https:// jobs.apple.com/search?job=73298437\&openJobId=73298437\#\&openJobId=73298437>.

27. LinkedIn (2017): Translation Project Coordinator - Barcelona. LinkedIn. Consultado el 19 de junio de 2017, <https://www.linkedin.com/jobs/view/365325765/>.

28. Lion People (2017): London- Strong Project Manager and Client Facing! Large LSP. Lion People. Consultado el 30 de octubre de 2016, <https://lionpeopleglobal.com/job/london-strong-projectmanager-and-client-facing-large-lsp/>.

29. SAP (2017): iXp Intern, Translation Project Coordinator Job. SAP. Consultado el 13 de mayo de 2017, <https://jobs.sap.com/job/Vancouver-iXp-Intern\%2C-Translation-Project-Coordinator-JobBC/341595001/?feedId=194801\&utm_source=LILimitedListings\&utm_campaign =SAP_LL $>$.

30. Idem Translations, Inc. (2017): Localization Project Manager. LinkedIn. Consultado el 7 de mayo de 2017, <https://www.linkedin.com/jobs/view/320767549/?recommendedFlavor=IN_NETWOR K\&refId=2561008671494153527143\&trk=d_flagship3_search_srp_jobs $>$.

\section{REFERENCIAS BIBLIOGRÁFICAS}

Ahsan, Kamrul, Ho, Marcus, Khan, Sabik, et al. (2013): Recruiting Project Managers: A Comparative Analysis of Competencies and Recruitment Signals from Job Advertisements. Project Management Journal. 44(5):36-54.

Arevalillo Doval, Juan-José (2002): Y tú, ¿traduces o proyectas? La linterna del traductor. 2:11-18.

Arevalillo Doval, Juan-José (2016): Gestión de proyectos, control de calidad y categorización de errores a partir del proceso de revisión. Tesis de doctorado no publicada. Málaga: Universidad de Málaga.

Bowker, Lynne (2004): What Does It Take to Work in the Translation Profession in Canada in the $21^{\text {st }}$ Century? Exploring a Database of Job Advertisements. Meta. 49(4):960-972.

Calvo Encinas, Elisa (2010): Análisis curricular de los estudios de Traducción e Interpretación en España: perspectiva del estudiantado. Tesis de doctorado no publicada. Granada: Universidad de Granada.

Chan, Andy Lung Jan (2008): Information Economics, the Translation Profession and Translator Certification. Tesis de doctorado no publicada. Tarragona: Universidad Rovira i Virgili.

Chesterman, Andrew (2009): The Name and Nature of Translator Studies. Hermes Journal of Language and Communication Studies. 42:14-20.

Dunne, Elena S. (2013): Project Risk Management: Developing a Risk Framework for Translation Projects. Tesis de doctorado no publicada. Kent: Kent State University.

Dunne, Keiran J. y Dunne, Elena S., eds. (2011): Translation and Localization Project Management: The Art of the Possible. Amsterdam/Philadelphia: John Benjamins.

Esselink, Bert (2000): A Practical Guide to Localization. Amsterdam/Philadelphia: John Benjamins.

Ferrer Simó, María R. (2016): La gestión de proyectos de traducción audiovisual en España. Seis estudios de caso. Tesis de doctorado no publicada. Castellón: Universitat Jaume I. 
Flores AcuÑA, Estefanía (2012): Gestión de proyectos de traducción en el aula: una experiencia en la clase de Traducción especializada italiano/español/italiano. UPO Innova. Revista de Innovación Docente. 1:191-204.

Gouadec, Daniel (2002): Profession: Traducteur. Paris: La Maison du Dictionnaire.

Gouadec, Daniel (2005): Modélisation du processus d'exécution des traductions. Meta. 50(2):643-655.

Gouadec, Daniel (2007): Translation as a Profession. Amsterdam/Philadelphia: John Benjamins.

GouAdec, Daniel (2009): Guide des métiers de la traduction-localisation et de la communication multilingue et multimédia. Paris: La Maison du Dictionnaire.

HöLzLE, Katharina (2010): Designing and Implementing a Career Path for Project Managers. International Journal of Project Management. 28(8):779-786.

Hurtado Albir, Amparo, ed. (2017): Researching Translation Competence by PACTE Group. Amsterdam/Philadelphia: John Benjamins.

Johnson, Alonzo, Winter, Paul A., Reio, Thomas G. (Jr), et al. (2008): Managerial Recruitment: The Influence of Personality and Ideal Candidate Characteristics. Journal of Management Development. 27(6):631-648.

Kelly, Dorothy (2005): A Handbook for Translator Trainer. Manchester: St. Jerome.

López Noguero, Fernando (2002): El análisis de contenido como método de investigación. XXI. Revista de Educación. 4:167-179.

Mатсна Авомво, Joel-Emmanuel (2015): La gestión de proyectos (project management) y su implementación en los estudios de Traducción e Interpretación. Tesis de doctorado no publicada. Madrid: Universidad de Alcalá.

Matis, Nancy (2010): Comment gérer vos projets de traduction. Liège: Edi.pro.

Plaza-Lara, Cristina (2016): Integración de la competencia instrumental-profesional en el aula de traducción. Berlín: Frank\&Timme.

Project Management Institute (2002/2007): Project Management Competency Development $(P M C D)$ Framework. $2^{\text {nd }}$ ed. Newtown Square: Project Management Institute.

Project Management Institute (1996/2013): A Guide to the Project Management Body of Knowledge (PMBOK ${ }^{\oplus}$ Guide). $5^{\text {th }}$ ed. Newtown Square: Project Management Institute.

Pyм, Anthony (2009): Humanizing Translation History. Hermes Journal of Language and Communication Studies. 42:23-48.

Rico Pérez, Celia (2002): Translation and Project Management. Translation Journal. 6(4). Consultado el 30 de julio de 2017, <http://translationjournal.net/journal/22project.htm>.

Torres Hostench, Olga (2002): La gestió de projectes de localització de programari: principis estratègics per a l'elaboració del model genèric de procés de gestió del projecte. Tradumàtica. Traducció i Tecnologies de la Informació i la Comunicació. 1:1-6. Consultado el 30 de julio de 2017, <https://ddd.uab.cat/pub/tradumatica/15787559n1/15787559n1a2torres.pdf >.

WAY, Catherine (2009): Bringing Professional Practices into Translation Classrooms. In: Ian Kemble, ed. The Changing Face of Translation. Portsmouth: University of Portsmouth, 131-142.

Zouncourides-Lull, Alexandra (2011): Applying PMI Methodology to Translation and Localization Projects. Project Integration Management. In: Keiran J. Dunne y Elena S. Dunne, eds. Translation and Localization Project Management: The Art of the Possible. Amsterdam/Philadelphia: John Benjamins, 71-95. 